\title{
Demência secundária à lesão cerebral traumática em região frontotemporal: um relato de caso
}

\author{
Secondary dementia to traumatic brain injury in the frontotemporal region: \\ a case report
}

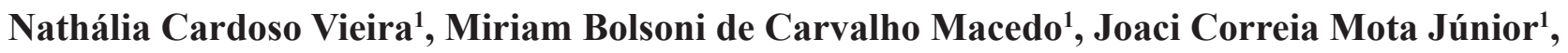
Letícia Carvalho Tiraboschi ${ }^{2}$ Tatiane Gomes da Silva Oliveira ${ }^{3}$
\end{abstract}

\begin{abstract}
Vieira NC, Macedo MBC Mota Júnior JC, Tiraboschi LC, Oliveira TCS. Demência secundária à lesão cerebral traumática em região frontotemporal: um relato de caso / Secondary dementia to traumatic brain injury in the frontotemporal region: a case report. Rev Med (São Paulo). 2020 jul.-ago.;99(4):394-9.
\end{abstract}

RESUMO: Introdução: A demência caracteriza-se pela deficiência cognitiva persistente que influencia negativamente as atividades diárias do paciente, podendo ser classificada em primária ou secundária a depender da etiologia. Diante dos conflitos diagnósticos na clínica médica atual e devido aos vários subtipos demenciais existentes, descrevemos um estudo de caso sobre uma Síndrome Demencial de etiologia incerta de um paciente em faixa etária pré-senil. Métodos: Foram realizadas três consultas ambulatoriais no período de quatro meses, com a realização do Mini Exame do Estado Mental (MEEM) e da Avaliação Cognitiva Montreal (MOCA), além de exames laboratoriais e de imagem para a elucidação do caso. Relato de Caso: Homem de 47 anos, com antecedentes pessoais de etilismo crônico por 35 anos, de tabagismo, de hipertensão arterial sistêmica e de traumatismo crânio encefálico (TCE) em região frontotemporal em 2006, desenvolveu transtornos neurocognitivos. Discussão: Na avaliação dessa síndrome demencial, procedemos com investigação clínica e complementar, em busca do diagnóstico e tratamento, na qual a história pregressa do paciente levou às hipóteses diagnósticas. Considerações finais: De acordo com os resultados dos exames, observou prejuízo da função cognitiva e redução volumétrica de massa encefálica após o TCE, obtendo melhora significativa após uso de Trazodona por doze meses.

Descritores: Demência/diagnóstico; Transtornos neurocognitivos/ diagnóstico.
ABSTRACT: Introduction: Dementia is characterized by persistent cognitive impairment which negatively influences the patient's daily activities and it can be classified as primary or secondary depending on the etiology. Given the diagnostic conflicts in the current medical clinic and due to the various existing dementia subtypes, we describe a case study about a dementia syndrome of uncertain etiology of a presenile age patient. Method: Three outpatient consultations were conducted over a period of four months, with the Mini Mental State Examination (MMSE) and the Montreal Cognitive Assessment (MOCA), as well as laboratory and imaging tests to elucidate the case. Case Report: A 47-year-old man with a 3-year history of chronic alcoholism, of smoking, of systemic arterial hypertension and of head trauma in the frontotemporal region in 2006, developed neurocognitive disorders. Discussion: In the evaluation of this demencial syndrome, we proceed with clinical and complementary insvestigation, in search of diagnosis and treatment, which the patient's historic led to diagnostics hypotheses. Final Remarks: According to the exams results, it was observed impairment of cognitive function, and volumetric reduction of brain mass after the head trauma, and obtained significative improvement after taking Trazodona for 12 months.

Keywords: Dementia/diagnosis; Neurocognitive disorders/ diagnosis.

Todos autores contribuíram igualmente para a pesquisa.

1. Acadêmica do curso de Medicina da Faculdade Morgana Potrich - FAMP, Mineiros, Goiás. ORCID: Vieira NC - https://orcid.org/0000-0002-8871992X; Macedo MBC - https://orcid.org/0000-0003-2995-146X; Mota Júnior JCM - https://orcid.org/0000-0001-9608-5915. E-mail: nathalia.sma86@ gmail.com; miimacedo@hotmail.com; joaci.mota27@gmail.com.

2. Graduada em Medicina pela Universidade Federal de Uberlândia, MG. Especialista em Psiquiatria pela Universidade de São Paulo de Ribeirão Preto, SP. Docente da Faculdade Morgana Potrich - FAMP, Mineiros, Goiás. ORCID: https://orcid.org/0000-0003-0670-1820. E-mail: leticiacarvalho@ fampfaculdade.com.br.

3. Graduada em Licenciatura Plena em Letras pela Universidade Estadual do Mato Grosso -UNEMAT, Especialista em Educação Inclusiva e LIBRAS pelo Instituto Federal de Mato Grosso - IFMT e Mestre em Linguística pela Universidade Estadual do Mato Grosso -UNEMAT. Docente da Faculdade Morgana Potrich - FAMP, Mineiros, Goiás. ORCID: https://orcid.org/0000-0003-1661-4085. E-mail: tatianeoliveira@fampfaculdade.com.br.

Endereço para correspondência: Nathália Cardoso Vieira. Avenida A, Quadra B2, lote 3-7, número 60, Apart. 212, bloco B, Setor Leste Vila Nova Residencial Vila Rica. Goiânia, Goiás. CEP: 74645-210. Email: nathalia.sma86@gmail.com. 


\section{INTRODUÇÃO}

síndrome demencial compreende uma clínica de alterações duradouras das capacidades cognitivas do paciente, a qual leva ao acometimento prejudicial das atividades diárias. Dentre as funções cognitivas que estão deficitárias encontra-se a memória, afecção mais bem vista e identificada, além da linguagem, orientação em tempo e espaço e funções executivas, por exemplo, planejamento de ações, hábitos do cotidiano e tomada de decisões ${ }^{1}$.

Sobre a prevalência global de demência, um estudo revelou que 24,3 milhões de pessoas têm demência hoje, com 4,6 milhões de novos casos por ano (um novo caso a cada 7 segundos), sendo que a maioria delas vivem nos países em desenvolvimento (60\% em 2001, aumentando para $71 \%$ em 2040) $)^{2}$.

De acordo com o Manual Diagnóstico e Estatístico de Transtornos Mentais - DSM- $5^{3}$, a demência, referida como transtornos neurocognitivos maiores, representa uma patologia complexa de estabelecer diagnóstico devido à existência de inúmeros subtipos, dentre eles: Demência Frontotemporal, Vascular, de Corpos de Lewy, induzido por substâncias, Doença de Alzheimer, Lesão Cerebral Traumática, entre outros.

Sendo assim, a variabilidade do diagnóstico de demência, no qual múltiplas etiologias podem coexistir, podendo prolongar o processo de decisão diagnóstica e/ ou tratamento para cada situação. Dessa forma, deve-se atentar primeiramente aos subdiagnósticos e, ainda, com as confusões diagnósticas entre os diversos subtipos da síndrome, evitando-se um vício frequente da prática clínica de se atribuir a toda e qualquer demência a etiologia de Alzheimer. Deve-se, inclusive, contemplar a realização de exames complementares para um diagnóstico diferencial ${ }^{4}$.

Devido as diversas causas existentes de demência, a pesquisa objetiva relatar o caso de um paciente com demência secundária à lesão cerebral traumática, por meio de acompanhamento a curto prazo, com avaliação de exames clínicos e complementares, conclui-se uma demência secundária à lesão cerebral traumática em região frontotemporal.

\section{MÉTODO}

Pesquisa amparada, do ponto de vista ético, pelo Comitê de Ética em Pesquisa, cujo Certificado de Apresentação para Apreciação Ética (CAAE) corresponde ao número 13309819.5.0000.5428. Trata-se de uma pesquisa descritiva, do tipo relato de caso, através do uso de fontes secundárias de dados por meio do prontuário (referente a primeira consulta de Psiquiatria em 2018, que despertou o interesse dos pesquisadores em relatar esse caso), após aceita no Comitê de Ética em Pesquisa. Além da aceitação do próprio paciente após o convite para participação da pesquisa, com assinatura do Termo de Consentimento Livre e Esclarecido (TCLE).

Foram realizadas três consultas ambulatoriais no período de quatro meses, com a realização do Mini Exame do Estado Mental (MEEM) e da Avaliação Cognitiva Montreal (MOCA), além de exames laboratoriais e de imagem para a elucidação do caso.

Todas as consultas foram realizadas no ambulatório de Psiquiatria, e os testes clínicos foram escolhidos por apresentarem maior facilidade de aplicação e por serem difundidos na área médica.

\section{RELATO DO CASO}

Paciente sexo masculino, 47 anos, ensino fundamental incompleto, realizou a primeira consulta de Psiquiatria em novembro do ano de 2018, acompanhado de três familiares, os quais relataram todos os acontecimentos e perguntas, visto que o paciente encontrava- se apático e com redução importante do fluxo de fala. A família relatou que, após um Traumatismo Crânio-Encefálico (TCE) em 2006 devido uma queda de moto com capacete e estando embriagado, ele teve perda de consciência seguida de hospitalização, e após 30 dias realizou a trepanação em região frontotemporal. Posteriormente o paciente começou a apresentar alteração do comportamento, tais como: esquecimentos, tremor (e flapping), insônia, irritabilidade, nervosismo e explosividade. Semanas antes à consulta, apresentou episódios de alucinações (vendo formigas no canto da parede e um cachorro latindo no quarto).

Paciente possui Hipertensão Arterial Sistêmica há aproximadamente 25 anos - em uso de propranolol. Tabagista com consumo de aproximadamente 30 cigarros/ dia, etilismo crônico por 35 anos (cachaça, não soube informar quantidade, acredita que mais de uma garrafa ao dia), mas interrompeu o hábito em 2018.

Relatam que há dois anos teve suposta crise convulsiva, fazendo uso de Fenobarbital e abandonou por conta própria. Um dos familiares afirmou que o paciente teve quatro crises convulsivas desde 2006, mas nenhuma delas observadas pela família.

Em 2006, semana antes da cirurgia, fez uso de Risperidona, Ácido Valproico e Fenobarbital prescritos por um psiquiatra, por apresentar agitação extrema, fugas 
de casa, delírios de que possuía vacas e fazenda, e também era egoísta com comidas. A família negou taquilalia, pensamento acelerado, ou outros comemorativos de mania bipolar. Apresentava insônia e fazia uso de Diazepam. Nesse período, ficou em abstinência alcóolica por cerca de três anos, por conta do tratamento medicamentoso, porém após cessação dos mesmos retornou ao consumo de álcool.

$\mathrm{Na}$ história familiar, possui dois irmãos faleceram por suicídio, uma irmã com transtorno depressivo. Sem história de delírios na família.

No Exame do Estado Mental (EEM) da consulta de novembro de 2018, possuía aparência envelhecida, emagrecido, atitude colaborativa, lúcido, desorientado em tempo e espaço (relatava que estava no ano do acidente e que estava em sua cidade natal), humor eutímico, afeto congruente com o humor, sem alterações da sensopercepção, há prejuízo da memória recente e dificuldade de reter informações. Memória retrógrada preservada, presença de tremores grosseiros (tendo sido questionado abstinência alcóolica). Fala quantitativamente diminuída. Leve bradpsiquismo. Disartria leve. Pragmático. Crítica de morbidade parcial.

Como Hipótese Diagnóstica e Conduta da consulta de novembro de 2018, obteve-se Demência alcóolica, demência frontotemporal secundária ao TCE. Naltrexona para tratamento de transtorno por uso do álcool; Trazodona de liberação prolongada, visando melhora de sono e comportamental; solicitado Ressonância Magnética de crânio, exames laboratoriais (alterações foram tratadas posteriormente). Foi considerado introduzir Ácido Valproico e Inibidor Seletivo de Recaptação de Serotonina (ISRS) para melhora comportamental.

Paciente retornou à consulta, em agosto de 2019 , acompanhado de sua irmã, quando foi realizado o convite para participação da atual pesquisa, sendo então realizada a primeira consulta para a pesquisa. Apresentou melhora dos sintomas da primeira consulta, com ausência de delírios e irritabilidade. Cessou o consumo de álcool desde dezembro de 2018. Continuou fazendo uso das medicações Propranolol, Naltrexona e Trazodona. Relatou dificuldade no sono inicial e com despertar precoce, incapaz de pegar no sono novamente. Afirmou tabagismo (não soube relatar a quantidade, mas diminuída em relação ao ano anterior), fumando também a noite quando não consegue dormir e de manhã após beber café. Relatou que desde o início do uso dos medicamentos citados acima obteve melhora do relacionamento familiar (por exemplo, ajuda a irmã nas atividades domésticas). A irmã disse que "os medicamentos foram "um milagre" e expressou a vontade de não interromper o uso de nenhum deles" (-sic).

O paciente trouxe a Ressonância Nuclear Magnética
(Figura 1), sendo observado redução da massa encefálica, principalmente do lobo frontal (justificando as alterações do comportamento), lesão sequelar na porção posterior do tronco do corpo caloso, regiões de hipossinal sugestivas de lesão axonal traumática (justificado pelo TCE), além de não haver desvio da linha média, sem lesões com efeito de massa e tronco cerebral e pares cranianos sem alterações; e exames laboratoriais, como hemograma (apresentando leve anemia que foi tratada), valores dentro do esperado de TSH, T4 livre, glicemia em jejum, lipidograma, ácido úrico e creatinina, fosfatase alcalina e vitamina B1, além de valores elevados de vitamina B12 $(549,5 \mathrm{pg} / \mathrm{ml})$, gama GT (847 U/L), TGO (48 U/L), e TGP (52 U/L), primeiro resultado não justificado devido falta de informação sobre tratamento para anemia prévia, e os outros resultados se alteraram devido consumo excessivo de álcool. Exames esses realizados em novembro de 2018.

Na consulta realizada em agosto de 2019, foi feito o $\mathrm{MEEM}^{5}$, apresentando o resultado de 24 pontos totais (4 anos de estudo), perdeu 3 pontos em orientação de tempo e espaço, e 3 pontos em evocação, com demais preservados, concluindo, então, um grau de demência, visto que o ponto de corte para pacientes com até quatro anos de escolaridade é menor ou igual à 24 pontos.

No EEM da consulta de agosto de 2019, na consulta em consultório médico, acompanhado de sua irmã, observou aparência adequada com a idade cronológica, bom asseio pessoal, atitude colaborativa, lúcido, um pouco desorientado em tempo e espaço (não soube relatar o bairro que estava, o dia da semana e o mês), discurso confuso e algo inseguro, com risadas incompreensíveis quando se sentia incapaz de responder as perguntas propostas, humor eutímico, afeto congruente com o humor, sem alterações da sensopercepção, há prejuízo da memória remota (fatos ocorridos meses após o acidente).

Retorno para a segunda consulta da pesquisa em setembro de 2019, onde apresentava-se com história clínica igual da última consulta, fazendo uso regular dos medicamentos. Foi realizado Avaliação Cognitiva Montreal (MOCA) ${ }^{6}$, obtendo 17 pontos, indicando comprometimento cognitivo. Também foi realizado o Teste de Fagerstrom, obtendo resultado de 7 pontos (elevada dependência à nicotina), onde foi proposto terapia medicamentosa, paciente mostrou-se interessado, porém não pode acatar a esse tratamento no momento, devido condições financeiras.

$\mathrm{O}$ último retorno médico ocorreu em novembro de 2019 (terceira consulta), com apresentação de exames laboratoriais solicitados na última consulta para reavaliação do caso, onde observou-se melhora dos valores em relação aos relatados anteriormente, gama GT (19 U/L), TGO (19 $\mathrm{U} / \mathrm{L})$, e TGP (12 U/L). 

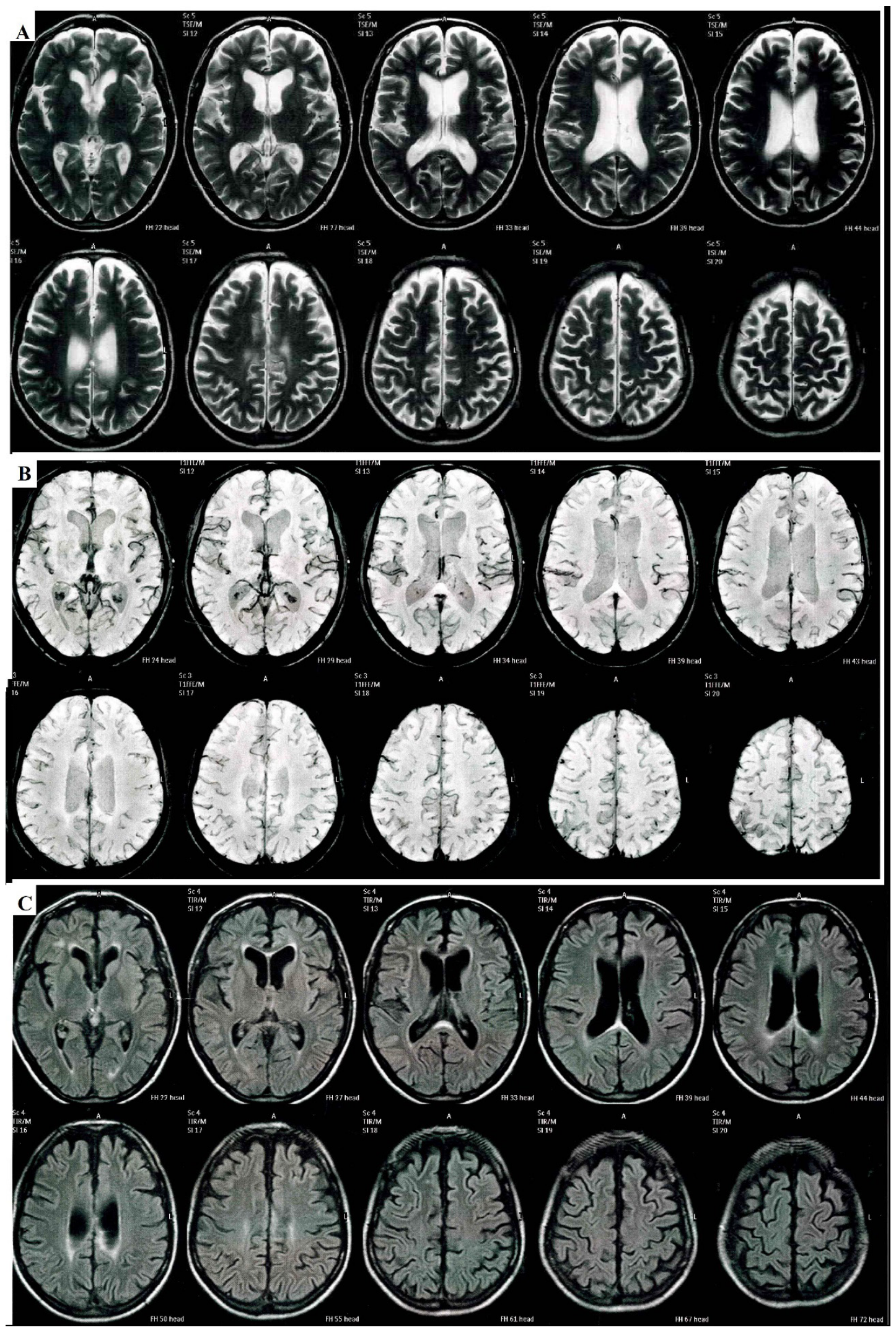

Figura 1: RNM realizada no dia 23 de novembro de 2018, sem uso de contraste. (A): corte sagital, mostrando pequena lesão sequelar no lobo temporal esquerdo, e lesão sequelar nas porções posteriores do corpo caloso; (B): corte axial em sequência SWI, evidenciando pequenas imagens focais de alteração do sinal, subcorticais na alta convexidade frontal bilateralmente, sugestivas de sequelas de lesão axonal traumática; (C): corte axial, com sinais de discreta redução volumétrica encefálica, notadamente nos lobos frontais 


\section{DISCUSSÃO}

O quadro demencial corresponde a alterações cognitivas do paciente, por exemplo, memória, linguagem, funções executivas, sendo que esse déficit apresente de maneira contínua e que influencie negativamente suas atividades diárias. Dessa forma, deve-se iniciar a investigação clínica e complementar para intervenção precoce do quadro ${ }^{7}$.

De acordo com o Manual Diagnóstico e Estatístico de Transtornos Mentais - DSM- $5^{3}$, para o diagnóstico de Demência devido a Lesão Cerebral Traumática é preciso de um ou mais dos itens: 1. Perda de consciência; 2. Amnésia pós-traumática; 3. Desorientação e confusão; 4. Sinais neurológicos (por neuroimagens), além de apresentar transtornos neurocogntivos imediatamente após a ocorrência do trauma. A partir da análise e acompanhamento do caso clínico em questão, o paciente contemplou todos os critérios, podendo chegar ao diagnóstico.

Estudos mostram que o TCE pode desencadear quadros demenciais em idade precoce, assim como gerar alterações cognitivas com gravidade relativamente maior em comparação as demências de curso normal, como o caso demostrado ${ }^{8}$.

É importante lembrar das características clínicas de um tipo de demência degenerativa, a Demência Frontotemporal (DFT), onde o doente irá apresentar alterações no comportamento, na fala, podendo até chegar a mutismo. O paciente em questão apresentava tais alterações o que se fez pensar em primeira análise nesse subtipo demencial, porém outra característica da DFT é a memória encontrar-se inalterada, o que gera um ponto negativo para essa hipótese diagnóstica, o que foi realmente descartado após investigações mais minuciosas ${ }^{9}$.

Manifestações comportamentais típicas de DFT como delírio, agressão, ansiedade e irritabilidade, tiveram um declínio considerável com o uso de $150 \mathrm{mg}$ de Trazodona, enquanto que com o uso de $300 \mathrm{mg}$ desta mesma substância se fizeram necessários em casos como depressão e comportamento motor aberrante. Resultados esses que também foram alcançados no tratamento do paciente em questão, que possuía como diagnóstico Demência secundária a Lesão Cerebral Traumática ${ }^{11}$.

Outra característica clínica do paciente relatado é o abuso de álcool por longo período de tempo, o que contribuiu para as alterações cognitivas do mesmo. É observado que os efeitos tóxicos do álcool podem causar comprometimento neurológico, devido atrofia de massa encefálica, podendo ser reversíveis após a cessação do consumo alcoólico. Outro ponto importante a salientar, é o fato de que o abuso de álcool pode ocasionar acidentes automobilísticos, causando TCE e gerando alterações neurocognitivas ${ }^{11}$.

Não foi encontrado na literatura tratamento da Demência secundária a Lesão Cerebral Traumática com uso de Trazodona, porém o presente relato de caso apresentou os efeitos benéficos da Trazodona na terapia medicamentosa dessa demência, visto que o paciente mudou completamente sua aparência física e comportamental comparando a consultas ocorridas com intervalo de um ano, estando mais compreensivo e colaborativo nas atividades diárias da família. O medicamento em questão, Trazodona, se trata de um agente serotonérgico atípico que tem como características a recaptação moderada de serotonina e um efeito antagonista serotoninérgico com um metabolito ativo meta-clorofenil-piperazina (m-CPP) ${ }^{12}$.

Dessa forma, o paciente em questão obteve boa melhora de comportamento e humor após introdução medicamentosa da Trazodona, porém ainda há prejuízo cognitivo, visto a partir dos resultados obtidos nos testes clínicos (MEEM, MOCA), principalmente no âmbito da memória e orientação em tempo e espaço. Apesar de não possuir testes anteriores para efeito comparativo, e possuir apenas quatro anos de escolaridade, para avaliar os benefícios da terapêutica aplicada, pode-se evidenciar uma melhora geral do quadro clínico, principalmente no âmbito afetivo do paciente.

\section{CONCLUSÃO}

Portanto, em virtude da investigação clínica e complementar, observou alterações cognitivas severas após TCE, com melhora quase total dos sintomas após uso de doze meses de Trazodona, e atual prejuízo significativo da função cognitiva, com obtenção de nota 24 no MEEM, e 17 pontos no MOCA, e redução volumétrica de massa encefálica notadamente em região frontal, além de lesão sequelar em região temporal e outras alterações traumáticas vistas na Ressonância Magnética realizada após o TCE sofrido pelo paciente, concluindo- se de uma demência secundária à lesão cerebral traumática em região frontotemporal.

Recomenda-se realizar um acompanhamento a longo prazo para caso semelhantes, se possível, para ser observado a evolução do paciente através de mais avaliações, aplicar de forma mensal os testes utilizados nessa pesquisa e, assim, consumar o diagnóstico. Além de se orientar uma terapia multidisciplinar, com envolvimento de psicólogo tanto para o paciente com quadro de demência quanto para os familiares que auxiliam no tratamento.

Participação dos autores: Vieira NC - planejamento do projeto, coleta e análise da história clínica, redação do texto. Macedo $M B C$ - planejamento do projeto, coleta e análise da história clínica, redação do texto. Mota Júnior JCM - planejamento do projeto, coleta e análise da história clínica, redação do texto. Tiraboschi $L C$ - orientações inicias, planejamento do projeto, coleta de dados clínicos e revisão do texto. Oliveira TGS - orientações estruturais, planejamento do projeto e revisão do texto. Para devidos fins, informamos que 
o artigo foi elaborado de forma conjunta entre os autores.

O Projeto deste artigo foi apresentado em pôster na X Mostra Científica da Faculdade Morgana Potrich (FAMP), 21 de outubro de 2019. Também foi inscrito e aprovado no Programa Institucional Voluntário de Iniciação Científica (PIVIC) - 2019/2020 da FAMP. E artigo utilizado como trabalho de conclusão de curso apresentado à FAMP, 5 de dezembro de 2019.

\section{REFERÊNCIAS}

1. Nitrini R, Caramelli P. Demências. In: Nitrini R, Bacheschi LA. A neurologia que todo médico deve saber. $2 \mathrm{a}$ ed. São Paulo: Atheneu; 2003. pt. 2, cap.15, p.323-34.

2. Forlenza OV, Aprahamian I, Perroco TB, Bottino CMC. Demências. In: Miguel EC, Gentil V, Gattaz WF, Bottino CMC, Tavares H, Forlenza OV, et al. Clínica psiquiátrica: a visão do Departamento e Instituto de Psiquiatria do HCFMUSP. Barueri, SP: Manole; 2011. Seção 5, cap.48, p. 582- 602 .

3. Jeste DV, Lieberman JA, Fassler D, Peele R, Benson S, Young ML, et al. Manual diagnóstico e estatístico de transtornos mentais. 5a ed. Porto Alegre: Artmed; 2014. p.602-4; 624-7.

4. Caramelli P, Barbosa MT. Como diagnosticar as quatro causas mais frequentes de demência? Rev Bras Psiquiatr. 2002;24:7-10. doi: http://dx.doi.org/10.1590/S151644462002000500003.

5. Biblioteca Virtual em Saúde. Atenção Primária em Saúde. Mini Exame do Estado Mental (MEEM). Disponível em: https://aps.bvs.br/apps/calculadoras/?page $=11$.

6. Universidade Federal de Santa Catarina. Montreal Cognitive Assessment (MOCA). Versão Experimental Brasileira. Disponível em: https://neurologiahu.ufsc.br/files/2012/09/ MoCA-Test-Portuguese_Brazil.pdf.
7. Vaconcellos AF, Silva DA, Machado DA, Franco TR, Moraes EM. Demência reversível: relato de caso. Braz Geriatr Gerontol. 2008;2(3):132-6. Disponível em: http://www. ggaging.com/how-to-cite/333/pt-BR.

8. Mendez MF. What is the relationship of traumatic brain injury to dementia? J Alzheimer's Dis. 2017;57(3):67-681. doi: https://doi.org/10.3233/JAD-161002.

9. Paiva GP, Maldonado FHR, Spaziani AO. Demência frontotemporal em paciente feminina de 56 anos: relato de caso. Arch Health Invest. 2019;8(3):125-9. doi: http://dx.doi. org/10.21270/archi.v8i3.3243.

10. Lebert F, Stekke W, Hasenbroekx C, Pasquier F. Frontotemporal dementia: a randomised, controlled trial with trazodone. Dement Geriatr Cogn Disord. 2004;17:355-9. doi: https:// doi.org/10.1159/000077171.

11. Haes TM, Clé DV, Nunes TF, Roriz-Filho JS, Moriguti JC. Álcool e sistema nervoso central. Medicina (Riberão Preto). 2010;43(2):153-63. Disponível em: http://revista.fmrp.usp. br/2010/vol43n2/Simp7 \%C11cool\%20e\%20sistema\%20 nervoso $\% 20$ central.pdf.

12. Lebert F. Behavioral benefits of trazodone are sustained for the long term in frontotemporal dementia. Therapy. 2006;39(1):936. doi: https://doi.org/10.1586/14750708.3.1.93.

Submetido: 12.12 .2019

Aceito: 29.05.2020 